\title{
An axiomatic re-characterization of the Kemeny rule
}

Citation for published version (APA):

Can, B., Pourpouneh, M., \& Storcken, T. (2022). An axiomatic re-characterization of the Kemeny rule. Review of Economic Design, 26(3), 447-467. https://doi.org/10.1007/s10058-021-00259-2

Document status and date:

Published: 01/09/2022

DOI:

10.1007/s10058-021-00259-2

Document Version:

Publisher's PDF, also known as Version of record

Document license:

Taverne

Please check the document version of this publication:

- A submitted manuscript is the version of the article upon submission and before peer-review. There can be important differences between the submitted version and the official published version of record.

People interested in the research are advised to contact the author for the final version of the publication, or visit the DOI to the publisher's website.

- The final author version and the galley proof are versions of the publication after peer review.

- The final published version features the final layout of the paper including the volume, issue and page numbers.

Link to publication

\footnotetext{
General rights rights.

- You may freely distribute the URL identifying the publication in the public portal. please follow below link for the End User Agreement:

www.umlib.nl/taverne-license

Take down policy

If you believe that this document breaches copyright please contact us at:

repository@maastrichtuniversity.nl

providing details and we will investigate your claim.
}

Copyright and moral rights for the publications made accessible in the public portal are retained by the authors and/or other copyright owners and it is a condition of accessing publications that users recognise and abide by the legal requirements associated with these

- Users may download and print one copy of any publication from the public portal for the purpose of private study or research.

- You may not further distribute the material or use it for any profit-making activity or commercial gain

If the publication is distributed under the terms of Article $25 \mathrm{fa}$ of the Dutch Copyright Act, indicated by the "Taverne" license above, 


\title{
An axiomatic re-characterization of the Kemeny rule
}

\author{
Burak Can ${ }^{1} \cdot$ Mohsen Pourpouneh ${ }^{2}$ (i) $\cdot$ Ton Storcken $^{3}$
}

Received: 30 March 2021 / Accepted: 3 August 2021

๑ The Author(s), under exclusive licence to Springer-Verlag GmbH Germany, part of Springer Nature 2021

\begin{abstract}
The Kemeny rule is one of the well studied decision rules. In this paper we show that the Kemeny rule is the only rule which is unbiased, monotone, strongly tiebreaking, strongly gradual, and weighed tournamental. We show that these conditions are logically independent.
\end{abstract}

Keywords Kemeny rule $\cdot$ Preference aggregation $\cdot$ Axiomatic characterization

JEL Classification D70 · D71 · D72

\section{Introduction}

Social choice rules are correspondences that assign sets of alternatives as collectively chosen outcomes to preference profiles of linear orders over a set of alternatives, while preference rules assign sets of linear orders as collectively chosen outcomes to these profiles. In each of these settings, a unanimous agreement, i.e., everyone

This work is mostly financed by the Netherlands Organisation for Scientific Research (NWO) under the grant with project no. 451-13-017 (VENI, 2014) and partially by Fonds National de la Recherche Luxembourg. The support of both institutes, therefore, is gratefully acknowledged.

This work is supported by the Center for Blockchains and Electronic Markets (BCM) funded by the Carlsberg Foundation under Grant No. CF18-1112.

$凶$ Mohsen Pourpouneh

mohsen@ifro.ku.dk

Burak Can

b.can@maastrichtuniversity.nl

Ton Storcken

t.storcken@maastrichtuniversity.nl

1 Department of Data Analytics and Digitalisation, Maastricht University, Maastricht, The Netherlands

2 Department of Food and Resource Economics (IFRO), University of Copenhagen, Copenhagen, Denmark

3 Department of Quantitative Economics, Maastricht University, Maastricht, The Netherlands 
having the same preference, would lead to a trivial outcome (a unanimous consensus alternative/preference) and any sensible choice/preference rule would behave this way.

The difference in rules stem from decisions in non-trivial cases. Social choice/ preference rules can behave very differently in cases where unanimity is not observed in the preference profiles. One way to still touch upon this unanimity idea is the following: the rules can still try to find the alternative/preference which is "closest" to being the unanimity winner ${ }^{1}$. When closeness is defined, for instance, by the Kemeny distance, we notice that Borda choice rule and Kemeny-Young $(1959,1978)$ preference rule are very similar, i.e., Borda (as a choice rule) chooses the alternative which is the "closest" to being the unanimity winner, while Kemeny (as a preference rule) chooses the linear order which is the "closest" to being the unanimity preference. ${ }^{3}$

This paper shows that despite the aforementioned conceptual similarities between Borda and Kemeny, there are still important axiomatic differences between these rules. One would expect that axiomatic similarities will carry over from Borda choice rule to Kemeny preference rule. Borda choice rule is known to be consistent (Young 1974), so is the Kemeny preference rule. However, as well-known, Borda fails Maskin (1999) monotonicity and so do many other scoring rules [exceptions are characterized by Doğan and Koray (2015)]. Despite this monotonicity failure in Borda choice rule, Can and Storcken (2013) has shown a monotonic characterization of the Kemeny preference rule. This paper proves that indeed monotonicity is a very concrete feature of the Kemeny (as a preference) rule, despite it's score-like algorithm and similarities to Borda (as a choice rule). We provide some novel and normative conditions on preference rules with which we prove a new monotonic characterization of the Kemeny preference rule. Thereafter, in Sect. 6 we re-visit some axiomatic features shared also by Borda and Kemeny (both as preference rules).

The set of characterizing conditions used in this paper are being unbiased, monotone, weighed tournamental, strongly tie-breaking, and strongly gradual. Being unbiased requires that for profiles at which all possible preferences are reported the same number of times, the outcomes must equal to all possible linear orders. Being (update) monotone requires that if an update occurs in one of the agent's preferences towards one of the outcomes, then this update should change the result in such a way that the new outcomes still contain this preference, and possibly more but no more than what was present before the update. ${ }^{4}$ In Can et al. (2021), being tournamental is defined as "outcomes should only depend on the tournament results on pairs of alternatives in the profiles". We use a weaker version of this condition, i.e., being weighed tournamental, which requires that outcomes depend also on the pairwise margins of these tournaments. ${ }^{5}$ Being strongly tie-breaking requires that if there's indecisiveness

\footnotetext{
1 In the domain of social choice rules, Nitzan (1981) introduced "Closeness to Unanimity Procedure" as a first example to distance rationalizability. approach ${ }^{2}$ and showed that the Borda (1784) rule is the closest to unanimity under the Kemeny (1959) distance.

3 Saari and Merlin (2000) characterize all single profile paradoxes and behavior of the Kemeny rule. Klamler (2004) compares the Kemeny rule with other distance based rules such as the Slater (1961) and the Dodgson (1876) rules. In terms of the computational efficiency of the Kemeny rule, see Endriss and de Haan (2015) and Conitzer (2006).

4 See Can and Storcken (2013).

5 See Sect. 6.2 for a discussion on these two conditions.
} 
over two alternatives, e.g., the outcomes comprise preferences with alternatives $a$ over $b$, and also those with $b$ over $a$, then any deviation in the profile concerning these alternatives should break this tie. Roughly speaking, being strongly tie-breaking captures the tie-breaking-part of positive responsiveness as introduced by May (1952). Finally, being strongly gradual requires that in case of an even number of agents, if the outcomes were decisive over two alternatives, e.g., all of them ranked $a$ over $b$, then no minor deviation concerning $a$ and $b$, can turn the outcomes into a decisive win for $b$ over $a$.

The paper is organized as follows. In Sect. 2 we formalize some basic notions and the model. The characterizing conditions are treated in Sect. 3 and in Sect. 4 we show how these translate to weighed tournament correspondences. Section 5 discusses that the Kemeny rule satisfies these conditions and that it is the only rule which does so. Section 6 discusses the independence of the conditions used and concludes the paper.

\section{Model basics}

Let $\mathbb{N}=\{1,2, \ldots\}$, the set of positive integers, denote the set of potential individuals. We take the first $n$ elements of $N=\{1, \ldots, n\}$ as the set of (concerned) agents. Let $A$ be a finite set of $m$ alternatives with $m \geq 3$. Let $|S|$ denote the cardinality of an arbitrary (finite) set $S$.

Let $\mathbb{L}$ denote the set of all possible linear orders over $A$. That is, the set of all weakly complete, irreflexive, asymmetric and transitive binary relations on $A$. For any $R \in \mathbb{L}$ and any two alternatives $a, b \in A$ we interpret $(a, b) \in R$ as $a$ is ordered above $b$ at $R$. In case there is no confusion, we suppress the parenthesis and write $a b \in R$ instead of $(a, b) \in R$. Similarly, $R=a b c$ denotes a linear order where $R=\{a b, a c, b c\}$. Let $-R$ denote the reverse of a linear order, formally $-R=\{y x: x y \in R\}$. For any subset $V$ of $\mathbb{L}$, let $V_{a b}$ denote the set of linear orders in $V$ in which $a$ is ordered strictly above $b$, formally $V_{a b}=\{R \in V: a b \in R\}$. For instance, we write $\mathbb{L}_{a b}$ to denote the set of all linear orders ordering $a$ above $b$.

Consider a set of agents $N$. A (preference) profile $p \in \mathbb{L}^{N}$ assigns to every agent $i \in N$, a linear order of $\mathbb{L}$. We denote the preference of an agent $i$ at a preference profile $p$ by $p(i)$. Therewith, for two different alternatives $a, b$ and an agent $i, a b \in p(i)$ means that agent $i$ (strictly) prefers $a$ to $b$ at the profile $p$. Hence, a profile represents a combination of individual preferences over the set of alternatives $A$, from all agents in $N$.

Let $N_{1}=\left\{1, \ldots, n_{1}\right\}$ and $N_{2}=\left\{1, \ldots, n_{2}\right\}$ be two sets of agents. Let $p \in \mathbb{L}^{N_{1}}$ and $q \in \mathbb{L}^{N_{2}}$ be two profiles. Then $p+q$ denotes the profile in $\mathbb{L}^{N_{3}}$, where $N_{3}=$ $\left\{1,2, \ldots, n_{1}+n_{2}\right\}$, defined for each agent $i \in N_{3}$ by

$$
(p+q)(i)=\left\{\begin{array}{cl}
p(i) & \text { if } \quad \\
q\left(i-n_{1}\right) & \text { if } i \in\left\{n_{1}+1, n_{1}+2, \ldots, n_{1}+n_{2}\right\}
\end{array} .\right.
$$

This formulation we call an addition of $p$ and $q$. Here the order in this "addition" matters as in general $p+q \neq q+p$. For a sequence of profiles, say $p^{1}$ up to $p^{k}$, the profile $p^{1}+\cdots+p^{k}$ is denoted by $\sum_{t=1}^{k} p^{t}$. Similarly, for a sequence of identical 
profiles such that for for all $t$, and for all agents $i \in N_{t}, p^{1}(i)=p^{2}(i)=\ldots=p^{k}(i)$, the profile $p^{1}+\cdots+p^{k}$ is denoted by $k \cdot p$ instead of $\sum_{t=1}^{k} p^{t}$.

Let $p$ be a preference profile over a set of $m$ alternatives. The $m \times m$ weighed tournament (matrix) $\bar{p}$ corresponding to profile $p$ is defined at an arbitrary entry $a b$, where $a$ and $b$ are alternatives, by

$$
\bar{p}_{a b}=\max \{|\{i: a b \in p(i)\}|-|\{i: b a \in p(i)\}|, 0\} .
$$

Hence, at entry $a b$, the value of $\bar{p}_{a b}$ is either strictly positive or zero. In the former case it indicates the numerical surplus of agents preferring $a$ to $b$ compared to those who prefer $b$ to $a$. In the latter case there is a (weak) majority preferring $b$ to $a$ in this binary comparison. Let $\mathbb{W}$ denote the set of all such $m \times m$ matrices. That is a $m \times m$ matrix where all cells have non-negative integers as entries and we have $\bar{p}_{a b}=0$ or $\bar{p}_{b a}=0$ for all $a b$. Given $m$, let $\mathbf{0}$ denote the weighed tournament where all the entries are zero. Let $T_{w}$ denote the (partial pairwise majority) tournament associated with the weighed tournament $w \in \mathbb{W}$ and is defined by $T_{w}=\left\{a b \in A \times A: w_{a b}>0\right\}$. That is, $T_{w}$ is the set of all distinct $a, b \in A$ such that $a$ beats $b$ by a strict majority in the weighed tournament $w$. When we consider the weighed tournament $\bar{p}$ corresponding to a profile $p$, we simply write $T_{p}$ instead of $T_{\bar{p}}$.

The Kemeny distance between two linear orders, $R$ and $R^{\prime}$, is defined by $\delta\left(R, R^{\prime}\right)=$ $\left|R \backslash R^{\prime}\right|+\left|R^{\prime} \backslash R\right|$. It is well-known that $\delta$ satisfies the regular conditions of being a metric. The extension of the Kemeny distance between a linear order $R$ and a profile $p$ in $\mathbb{L}^{N}$ is defined by $\delta(p, R)=\sum_{i \in N} \delta(p(i), R)$.

We consider preference rule, e.g., $\varphi$, which assign a non-empty subset of linear orders $\varphi(p)$ to every profile $p \in \mathbb{L}^{N}$ for every set of agents $N$. A rule aggregates the preferences of the agents into a set of linear orders $\varphi(p)$, as the collective set of outcomes at profile $p$. At any profile $p$, the Kemeny rule $\varphi^{K}(p)$ assigns the linear orders which minimizes the Kemeny distance to the preference profile. Formally,

$$
\varphi^{K}(p)=\left\{R \in \mathbb{L}: \delta(p, R) \leq \delta\left(p, R^{\prime}\right) \text { for all } R^{\prime} \in \mathbb{L}\right\}
$$

Can and Storcken (2013) and Young and Levenglick (1978) also show an equivalent formulation. In this interpretation, the Kemeny rule $\varphi^{K}(p)$ assigns the linear orders which minimize the weighed majority disagreements. Formally,

$$
\varphi^{K}(p)=\left\{R \in \mathbb{L}: \sum_{x y \in R} \bar{p}_{y x} \leq \sum_{x y \in R^{\prime}} \bar{p}_{y x} \text { for all } R^{\prime} \in \mathbb{L}\right\}
$$

Remark 1 In what follows, we will use both formulations of the Kemeny rule without further reference. Moreover, we introduce $\kappa(R, p)=\sum_{x y \in R} \bar{p}_{y x}$. It can be seen as the sum of "disagreement surpluses" with $R$ at profile $p$. We extend this definition of $\kappa$ to a weighed tournament $w$ and linear order $R$ as follows $\kappa(R, w)=\sum_{x y \in R} w_{y x}$. 


\subsection{Elementary changes}

We define the notion of the elementary change in linear orders, profiles and weighed tournaments. In words an elementary change is a "slightest" possible change in the domain.

Let $a$ and $b$ be two distinct alternatives in $A$. Let $R, R^{\prime}$ be two linear orders in $\mathbb{L}$. We say $\left(R, R^{\prime}\right)$ forms an elementary change from $a b$ to $b a$ whenever $R \backslash\{a b\}=$ $R^{\prime} \backslash\{b a\}$. Note that in that case $a$ is consecutively ordered above $b$ at $R$ and $b$ is so above $a$ at $R^{\prime}$, where going from $R$ to $R^{\prime}$ only the positions of $a$ and $b$ are swapped. Further, $\delta\left(R, R^{\prime}\right)=2$ which is the minimum distance for two different linear orders. For two profiles $p$ and $q$, both in $\mathbb{L}^{N}$, we say that $(p, q)$ forms an elementary change from $a b$ to $b a$ whenever there exist an agent $i$ such that $p(i)$ and $q(i)$ form an elementary change from $a b$ to $b a$ and $p(j)=q(j)$ for all $j \in N \backslash\{i\}$. So, at profiles elementary changes occur precisely when one agent swaps the ranking of two consecutively ordered alternatives and all other agents do not change their preferences. It therewith is the "slightest" possible change between different profile.

Let $v$ and $w$ be weighed tournaments in $\mathbb{W}$. We say that $(v, w)$ forms an elementary change from $a b$ to $b a$ whenever one of the following three holds

1. $v_{a b}=w_{a b}+2$ and $w_{x y}=v_{x y}$ for all entries $x y \neq a b$, or

2. $v_{b a}+2=w_{b a}$ and $w_{x y}=v_{x y}$ for all entries $x y \neq b a$, or

3. $v_{a b}=1, w_{b a}=1, v_{b a}=w_{a b}=0$, and $v_{x y}=w_{x y}$ for all other entries $x y \neq b a$ and $x y \neq a b$.

That is, the only difference between the two matrices is that either $v_{a b}$ is decreased by two, or $v_{b a}$ is increased by two, or $v_{a b}$ is decreased by one and $v_{b a}$ is increased by one when going from $v$ to $w$. Note that whenever profiles $(p, q)$ forms an elementary change form $a b$ to $b a$ then either

1. $\bar{p}_{a b}=\bar{q}_{a b}+2$ and $\bar{p}_{x y}=\bar{q}_{x y}$ for all other entries $x y \neq a b$, or

2. $\bar{p}_{b a}+2=\bar{q}_{b a}$ and $\bar{p}_{x y}=\bar{q}_{x y}$ for all other entries $x y \neq b a$, or

3. $\bar{p}_{a b}=1, \bar{q}_{b a}=1, \bar{p}_{b a}=\bar{q}_{a b}=0$, and $\bar{p}_{x y}=\bar{q}_{x y}$ for all other entries $x y \neq b a$ and $x y \neq a b$.

Note that in case, the number of agents is even then all entries in $\bar{p}$ are even the third case does not apply.

\subsection{Building blocks profiles}

McGarvey (1953), introduced the following two agent profiles, denoted by $r^{a b, R} \in$ $\mathbb{L}^{\{1,2\}}$. Let $a$ and $b$ be two distinct alternatives and $R$ be a linear order such that at $R, a$ is consecutively ordered above $b$. Let $r^{a b, R}(1)=R$ and $r^{a b, R}(2)=(-R \backslash\{b a\}) \cup\{a b\}$. That is at such profiles agent 1 and 2 only agree on pair $a b$. As an example, for linear order $R=a b c_{1} c_{2} \ldots c_{k}$, we have $r^{a b, R}(1)=a b c_{1} c_{2} \ldots c_{k}$ and $r^{a b, R}(2)=$ $c_{k} \ldots c_{2} c_{1} a b$. Note that, for such profiles the associated weighed tournament is $\bar{r}^{a b, R}{ }_{x y}=0$ if $x y \neq a b$ and $\bar{r}^{a b, R} a b=2$, and the corresponding tournament is $T_{r^{a b, R}}=\{a b\}$. In case the choice of $R$ is not important we may write $r^{a b}$ instead of 
$r^{a b, R}$. Let $w$ be an arbitrary weighed tournament. Then we can associate a profile $r^{w}$ defined by $r^{w}=\sum_{x y \in A \times A, x \neq y} w_{x y} \cdot r^{x y}$, to $w$. Clearly $\overline{r^{w}}=2 \cdot w$.

Example 1 Consider the set of alternatives $A=\{a, b, c\}$ and $N=\{1,2,3,4,5,6\}$ with the preference profile $p=(a b c, a b c, b c a, b c a, c a b, c a b)$. Now, $T_{p}=\{a b, b c$, $c a\}$ and $\bar{p}$ is as follows

$$
\bar{p}=\begin{gathered}
a \\
a \\
b \\
c
\end{gathered}\left(\begin{array}{lll}
0 & 2 & 0 \\
0 & 0 & 2 \\
2 & 0 & 0
\end{array}\right)
$$

Let $w$ be the following weighed tournament:

$$
w=\begin{array}{ccc}
a & b & c \\
a \\
b \\
c
\end{array}\left(\begin{array}{lll}
0 & 3 & 0 \\
0 & 0 & 0 \\
0 & 0 & 0
\end{array}\right)
$$

Note that $2 \cdot w=\overline{r^{w}}$. Then an associated profile $r^{w}=(a b c, c a b, a b c, c a b, a b c, c a b)$.

\section{Characterizing conditions}

The characterization of Kemeny rule is based on five conditions we impose on any preference rule $\varphi$. First, let us consider a profile in which all linear orders in $\mathbb{L}$ are reported an equal number of times. We call such profiles uniform, i.e., $p \in \mathbb{L}^{N}$ is uniform, if for all $R, R^{\prime} \in \mathbb{L}$, we have $|i \in N: p(i)=R|=\left|i \in N: p(i)=R^{\prime}\right|$. The first condition we introduce, is a very mild one which ensures that in extreme cases where all linear orders are reported by an equal number of agents, the rule is not biased towards any outcome and assigns every linear order.

- Unbiased: $\varphi$ is unbiased (at uniform profiles), if for all sets of agents $N$, and all uniform profiles $p \in \mathbb{L}^{N}$,

$$
\varphi(p)=\mathbb{L} .
$$

The following (update) monotone condition is introduced by Can and Storcken (2013) as one of the characterizing conditions of the Kemeny rule.

- Monotone: $\varphi$ is (update) monotone, if for all pairs of profiles $(p, q)$ forming an elementary change from $a b$ to $b a$, with $\varphi(p)_{b a} \neq \emptyset$,

$$
\varphi(p)_{b a} \subseteq \varphi(q) \subseteq \varphi(p)
$$

That is, if an agent swaps a pair of two consecutively ordered alternatives in line with one of the rankings in the outcome then this ranking should be in the outcome also at the new profile. Furthermore, the new outcome of the new profile must be a subset of the outcome of the old profile. 
In regular tournaments, we only consider whether an alternative wins over another by a majority. In weighed tournaments, however, we also consider the net margin of this majority winning. The following condition requires that the rule behaves identical for profiles having the same "weighed tournaments" or a scalar of one another.

- Weighed tournamental: $\varphi$ is weighed tournamental, if for all profiles $p$ and $q$, with $\bar{p}=k \bar{q}$ for some positive integer $k$,

$$
\varphi(p)=\varphi(q)
$$

The next condition ensures that existing ties over two alternatives are immediately broken when there is a single elementary change concerning these two alternatives in the profile. This is a slight variation of the tie-breaking condition introduced in Can et al. (2021) for characterizing the Slater rule. Formally:

- Strongly tie-breaking: $\varphi$ is strongly tie-breaking, if for all pairs of profiles $(p, q)$ forming an elementary change from $a b$ to $b a$ in $\mathbb{L}^{N}$,

$$
\text { if } \varphi(p)_{a b} \neq \emptyset \text { and } \varphi(p)_{b a} \neq \varnothing \text {, then either } \varphi(q)_{a b}=\emptyset \text { or } \varphi(q)_{b a}=\emptyset \text {. }
$$

The final condition ensures that in case of an even number of agents, if all outcomes decisively put an alternative over another, a single elementary change reversing these two alternatives in the profile, cannot reverse the ordering of these alternative in all outcomes. This is a slight variation of the graduality condition introduced in Can et al. (2021) for characterizing the Slater rule. Formally:

- Strongly gradual: $\varphi$ is strongly gradual, if for all pairs of profiles $(p, q)$ forming an elementary change from $a b$ to $b a$ in $\mathbb{L}^{N}$, with $|N|$ even,

$$
\text { if } \varphi(p)_{b a}=\emptyset \text {, then } \varphi(q)_{a b} \neq \emptyset \text {. }
$$

In case $|N|$ is odd, we can face situations in which elementary changes can lead to swings in the majority winnings between two alternatives. Therefore, this may lead to drastic changes in the outcomes, instead of "gradual" changes. Hence the condition is restricted to even number of agents, wherein such swings cannot occur.

Lemma 1 Kemeny rule $\varphi^{K}$ is unbiased, monotone, weighed tournamental, strongly tie-breaking, and strongly gradual.

Proof In the following we show that the Kemeny rule satisfies each of these conditions. Unbiased: To prove that the Kemeny rule is unbiased, let $p$ be a uniform profile. It is sufficient to prove that $\varphi^{K}(p)=\mathbb{L}$. Since $p$ is a uniform profile, all entries in $\bar{p}$ are zero. So, for all linear orders $R$ we have that $\kappa(R, p)=0$, which implies $\varphi^{K}(p)=\mathbb{L}$, by Remark 1 .

Monotone: In Can and Storcken (2013) it is shown that the Kemeny rule is monotone. Weighed tournamental: It follows from the definition of the Kemeny rule and Remark 1 , that the Kemeny rule is weighed tournamental. 
Strongly tie-breaking: To show that the Kemeny rule is strongly tie-breaking, for some distinct alternatives $a$ and $b$ let $(p, q)$ form an elementary change from $a b$ to $b a$ in $\mathbb{L}^{N}$. Furthermore, suppose $\varphi^{K}(p)_{b a}$ is non-empty. Let $R \in \varphi^{K}(p)$ with $b a \in R$. It is sufficient to prove that $\varphi^{K}(q)_{a b}$ is empty. To the contrary let $R^{\prime} \in \varphi^{K}(q)$ with $a b \in R^{\prime}$. We deduce a contradiction. As $(q, p)$ forms an elementary change from $b a$ to $a b$ and $R^{\prime} \in \varphi^{K}(q)_{a b}$, being monotone implies $R^{\prime} \in \varphi^{K}(p)$. So, $\kappa(R, p)=\kappa\left(R^{\prime}, p\right)$. As $(p, q)$ forms an elementary change in $\mathbb{L}^{N}$ from $a b$ to $b a$ it follows that either $\bar{q}_{a b}<\bar{p}_{a b}$ or $\bar{p}_{b a}<\bar{q}_{b a}$ and $\bar{q}_{x y}=\bar{p}_{x y}$ for all entries $x y$ other than $a b$ and $b a$. Therefore,

$$
\begin{aligned}
\kappa(R, q) & =\bar{q}_{a b}+\sum_{x y \in R \backslash\{a b, b a\}} \bar{q}_{y x} \\
& \leq \bar{p}_{a b}+\sum_{x y \in R \backslash\{a b, b a\}} \bar{p}_{y x} \\
& =\kappa(R, p) \\
& =\kappa\left(R^{\prime}, p\right) \\
& =\bar{p}_{b a}+\sum_{x y \in R^{\prime} \backslash\{a b, b a\}} \bar{p}_{y x} \\
& \leq \bar{q}_{a b}+\sum_{x y \in R^{\prime} \backslash\{a b, b a\}} \bar{q}_{y x} \\
& =\kappa\left(R^{\prime}, q\right) .
\end{aligned}
$$

As at least one of these latter two weak inequalities is strict, $\kappa(R, q)<\kappa\left(R^{\prime}, q\right)$. But this contradicts that $R^{\prime} \in \varphi^{K}(q)$. This contradiction proves that $\varphi^{K}(q)_{a b}$ is empty.

Strongly gradual: To show that the Kemeny rule is strongly gradual, let $a$ and $b$ be distinct alternatives and let $p$ and $q$ be profiles in $\mathbb{L}^{N}$, such that $|N|$ is even and $(p, q)$ forms an elementary change from $a b$ to $b a$. Suppose $\varphi^{K}(p)_{b a}=\varnothing$. It is sufficient to prove that $\varphi^{K}(q)_{a b} \neq \emptyset$. As $\varphi^{K}(q)$ is the union of disjoint subsets $\varphi^{K}(q)_{a b}$ and $\varphi^{K}(q)_{b a}$, it is sufficient to prove that $\varphi^{K}(q)_{b a}$ is empty. Let $R^{1} \in \varphi^{K}(p)$, and to the contrary suppose $R^{2} \in \varphi^{K}(q)_{b a}$. As $\varphi^{K}(p)_{b a}=\emptyset$, we have $R^{1} \in \varphi^{K}(p)_{a b}$. Because $(p, q)$ forms an elementary change from $a b$ to $b a$, we have $\bar{p}_{x y}=\bar{q}_{x y}$ for all $x y$ other than $a b$ or $b a$. So,

$$
\begin{aligned}
& \alpha^{1}=\sum_{x y \in R^{1} \backslash\{a b, b a\}} \bar{p}_{y x}=\sum_{x y \in R^{1} \backslash\{a b, b a\}} \bar{q}_{y x}, \\
& \alpha^{2}=\sum_{x y \in R^{2} \backslash\{a b, b a\}} \bar{p}_{y x}=\sum_{x y \in R^{2} \backslash\{a b, b a\}} \bar{q}_{y x} .
\end{aligned}
$$

As $|N|$ is even all the entries $\bar{p}_{x y}$ and $\bar{q}_{x y}$ are even and so are $\alpha^{1}$ and $\alpha^{2}$. Because $(p, q)$ forms an elementary change from $a b$ to $b a$, we have $\bar{p}_{a b}=\bar{q}_{a b}+2$ and $\bar{p}_{b a}=$ $\bar{q}_{b a}=0$ or $\bar{p}_{b a}+2=\bar{q}_{a b}$ and $\bar{p}_{a b}=\bar{q}_{a b}=0$. We distinguish two cases accordingly.

Case $1 \bar{p}_{a b}=\bar{q}_{a b}+2$ and $\bar{p}_{b a}=\bar{q}_{b a}=0$. Now $\kappa\left(R^{1}, p\right)=\alpha^{1}$ and $\kappa\left(R^{2}, p\right)=$ $\alpha^{2}+\bar{p}_{a b}, \kappa\left(R^{1}, q\right)=\alpha^{1}$ and $\kappa\left(R^{2}, q\right)=\alpha^{2}+\bar{q}_{a b}$. As $R^{1} \in \varphi^{K}(p) \backslash \varphi^{K}(q)$ and $R^{2} \in \varphi^{K}(q) \backslash \varphi^{K}(p)$ we have $\kappa\left(R^{1}, p\right)<\kappa\left(R^{2}, p\right)$ and $\kappa\left(R^{2}, q\right)<\kappa\left(R^{1}, q\right)$. So, 


$$
\alpha^{1}<\alpha^{2}+\bar{p}_{a b} \text { and } \alpha^{2}+\bar{q}_{a b}<\alpha^{1} .
$$

Given that all the variables in the foregoing two inequalities are even these two inequalities can be rewritten as

$$
\alpha^{1}+2 \leq \alpha^{2}+\bar{p}_{a b} \text { and } \alpha^{2}+\bar{q}_{a b}+2 \leq \alpha^{1} .
$$

Adding these two inequalities and simplification yields

$$
\bar{q}_{a b}+4 \leq \bar{p}_{a b} .
$$

This yields a contradiction with the case assumption.

Case $2 \bar{p}_{b a}+2=\bar{q}_{a b}$ and $\bar{p}_{a b}=\bar{q}_{a b}=0$. This case is similar to the previous case.

\section{Weighed tournament correspondences}

In Sect. 5 we show that the Kemeny rule is the only rule which satisfies the conditions laid out in the previous section. To do this, we use "weighed tournament correspondences" which assign a non-empty set of linear orders to every weighed tournament.

Take any preference rule that is weighed tournamental. Then by construction, we can find an associated weighed tournament correspondence such that the outcome of the preference rule equals the outcome of this tournament correspondence. Since the weighed tournament correspondence is associated with the preference rule, it also inherits some of its properties. Therefore, we can abstract from profiles and base our analysis on weighed tournaments.

Let $2 \mathbb{W}$ denote the set of all weighed tournaments having all entries even, i.e., $2 \mathbb{W}=\{2 w: w \in \mathbb{W}\}$. A weighed tournament correspondence, say $\Phi$, assigns a non-empty subset of $\mathbb{L}$ to every weighed tournament $w$ in $2 \mathbb{W}$.

Correspondingly, let $\varphi$ be a preference rule which is weighed tournamental. With the construction laid out in Sect. 2.2, we can associate a weighed tournament correspondence $\Phi^{\varphi}$ such that $\Phi^{\varphi}$ assigns a subset of $\mathbb{L}$ to any arbitrary weighed tournament $w$ in $2 \mathbb{W}$ as follows:

$$
\Phi^{\varphi}(w)=\varphi(p) \text {, where } p \text { is a profile such that } \bar{p}=\alpha w \text { for some integer } \alpha \geq 1
$$

Next, we introduce some conditions on weighed tournament correspondences, inspired by those on preference rules discussed in Sect. 3. Then, in Lemma 2, we that given a weighed tournamental preference rule $\varphi$, any associated weighed tournament correspondence, say $\Phi^{\varphi}$, will "inherit" these conditions.

- W-unbiased: $\Phi$ is $\mathrm{W}$-unbiased if $\Phi(\mathbf{0})=\mathbb{L}$.

- W-monotone: $\Phi$ is $\mathrm{W}$-monotone, if for all weighed tournament pairs $\left(w, w^{\prime}\right)$ forming an elementary change in $2 \mathbb{W}$ from $a b$ to $b a$, with $\Phi(w)_{b a} \neq \emptyset$,

$$
\Phi(w)_{b a} \subseteq \Phi\left(w^{\prime}\right) \subseteq \Phi(w) .
$$


- W-tie-breaking: $\Phi$ is $\mathrm{W}$-tie-breaking, if for all weighed tournament pairs $\left(w, w^{\prime}\right)$ forming an elementary change in $2 \mathbb{W}$ from $a b$ to $b a$,

if $\Phi(w)_{a b} \neq \emptyset$ and $\Phi(w)_{b a} \neq \emptyset$, then either $\Phi\left(w^{\prime}\right)_{a b}=\emptyset$ or $\Phi\left(w^{\prime}\right)_{b a}=\emptyset$.

- $W$-graduality: $\Phi$ is $W$-gradual, if for all weighed tournament pairs $\left(w, w^{\prime}\right)$ forming an elementary change in $2 \mathbb{W}$ from $a b$ to $b a$,

$$
\text { if } \Phi(w)_{b a}=\emptyset \text {, then } \Phi\left(w^{\prime}\right)_{a b} \neq \emptyset \text {. }
$$

The following lemma shows that the weighed tournament correspondence $\Phi^{\varphi}$ inherits these " $W$ " properties from the weighed tournamental preference rule $\varphi$.

Lemma 2 (Inheritance Lemma) Let $\varphi$ be a weighed tournamental rule. Let $\varphi$ be unbiased, monotone, strongly tie-breaking and strongly gradual. Then $\Phi^{\varphi}$ is W-unbiased, $W$-monotone, W-tie-breaking and W-gradual.

Proof In the following we show that the associated weighed tournament rule $\Phi^{\varphi}$ satisfies these four conditions.

$W$-unbiased: Let $p$ be a uniform profile. Then, $\bar{p}=\mathbf{0}$. As $\varphi$ is unbiased, $\varphi(p)=\mathbb{L}$. Therefore by the definition of $\Phi^{\varphi}$ we have $\Phi^{\varphi}(\mathbf{0})=\mathbb{L}$. This proves that $\Phi^{\varphi}$ is Wunbiased.

The proof that $\Phi^{\varphi}$ is $\mathrm{W}$-monotone, W-tie-breaking and $\mathrm{W}$-gradual involves an elementary change, say $\left(w, w^{\prime}\right)$, from $a b$ to $b a$ for some distinct alternatives $a$ and $b$ and for some weighed tournaments $w$ and $w^{\prime}$ in $2 \mathbb{W}$. Define profiles $p^{\prime}$ and $q^{\prime}$ as follows

$$
\begin{aligned}
& p^{\prime}=\left(\sum_{x y \in A \times A, x \neq y, x y \notin\{a b, b a\}} \frac{1}{2} w_{x y} \cdot r^{x y, R^{x y}}\right)+\left(\frac{1}{2} w_{a b} \cdot r^{a b, R^{a b}}\right)+\left(\frac{1}{2} w_{b a} \cdot r^{b a, R^{b a}}\right) \text { and } \\
& q^{\prime}=\left(\sum_{x y \in A \times A, x \neq y, x y \notin\{a b, b a\}} \frac{1}{2} w_{x y}^{\prime} \cdot r^{x y, R^{x y}}\right)+\left(\frac{1}{2} w_{a b}^{\prime} \cdot r^{a b, R^{a b}}\right)+\left(\frac{1}{2} w_{b a}^{\prime} \cdot r^{b a, R^{b a}}\right) .
\end{aligned}
$$

Here for every $x y \in A \times A$, with $x \neq y, R^{x y}$ is a fixed linear order in both the expressions and taken such that $x$ is consecutively ordered above $y$. As $w$ and $w^{\prime}$ are in $2 \mathbb{W}$ and $\left(w, w^{\prime}\right)$ is an elementary change from $a b$ to $b a$, we have either

1. $w_{a b}=w_{a b}^{\prime}+2$ and $w_{x y}=w_{x y}^{\prime}$ for all entries $x y \neq a b$, or

2. $w_{a b}^{\prime}=w_{a b}+2$ and $w_{x y}=w_{x y}^{\prime}$ for all entries $x y \neq a b$.

Consider profiles $u^{a b}$ and $u^{b a}$ in $\mathbb{L}^{\{1,2\}}$, where $u^{a b}(1)=R^{a b}, u^{a b}(2)=-R^{a b}$, $u^{b a}(1)=R^{b a}$, and $u^{b a}(2)=-R^{b a}$. In case 1 take $p=p^{\prime}$ and $q=q^{\prime}+u^{a b}$ and in case 2 take $p=p^{\prime}+u^{b a}$ and $q=q^{\prime}$. By construction $p$ and $q$ are profiles in, say $\mathbb{L}^{N}$, with $|N|$ is even. Also by construction $(p, q)$ forms an elementary change from $a b$ to $b a, \bar{p}=w$, and $\bar{q}=w^{\prime}$. So, $\Phi^{\varphi}(w)=\varphi(p)$ and $\Phi^{\varphi}\left(w^{\prime}\right)=\varphi(q)$.

$W$-monotone: Take $a, b, w, w^{\prime}, p$, and $q$ as above. Suppose $\Phi^{\varphi}(w)_{b a} \neq \emptyset$. In order to prove that $\Phi^{\varphi}$ is $\mathrm{W}$-monotone it is sufficient to prove

$$
\Phi^{\varphi}(w)_{b a} \subseteq \Phi^{\varphi}\left(w^{\prime}\right) \subseteq \Phi^{\varphi}(w)
$$


As $\Phi^{\varphi}(w)=\varphi(p)$ we have $\varphi(p)_{a b} \neq \emptyset$. Because $\varphi$ is monotone this implies

$$
\varphi(p)_{b a} \subseteq \varphi(q) \subseteq \varphi(p)
$$

But $\Phi^{\varphi}(w)=\varphi(p)$ and $\Phi^{\varphi}\left(w^{\prime}\right)=\varphi(q)$ the former two inclusions follow by the latter two.

$W$-tie-breaking: Take $a, b, w, w^{\prime}, p$, and $q$ as above. Suppose $\Phi^{\varphi}(w)_{a b} \neq \emptyset$ and $\Phi^{\varphi}(w)_{b a} \neq \emptyset$. In order to prove that $\Phi^{\varphi}$ satisfies W-tie-breaking, it is sufficient to prove

$$
\Phi^{\varphi}\left(w^{\prime}\right)_{a b}=\emptyset \text { or } \Phi^{\varphi}\left(w^{\prime}\right)_{b a}=\varnothing .
$$

Because $\Phi^{\varphi}(w)=\varphi(p)$ it follows that both $\varphi(p)_{a b}$ and $\varphi(p)_{b a}$ are non-empty. As $\varphi$ is strongly tie-breaking this yields that either $\varphi(q)_{a b}=\varnothing$ or $\varphi(q)_{b a}=\varnothing$. Using $\Phi^{\varphi}\left(w^{\prime}\right)=\varphi(q)$ we have either $\Phi^{\varphi}\left(w^{\prime}\right)_{a b}=\emptyset$ or $\Phi^{\varphi}\left(w^{\prime}\right)_{b a}=\emptyset$.

$W$-graduality: Take $a, b, w, w^{\prime}, p$, and $q$ as above. Suppose $\Phi^{\varphi}(w)_{b a}=\emptyset$. In order to prove that $\Phi^{\varphi}$ is W-gradual it is sufficient to prove $\Phi^{\varphi}\left(w^{\prime}\right)_{a b} \neq \emptyset$. As $\Phi^{\varphi}(w)_{b a}=\varnothing$ and $\Phi^{\varphi}(w)=\varphi(p)$, it follows that $\varphi(p)_{b a}=\emptyset$. Because $\varphi$ is strongly gradual this implies $\varphi(q)_{a b} \neq \emptyset$. As $\varphi(q)=\Phi^{\varphi}\left(w^{\prime}\right)$ we have $\Phi^{\varphi}\left(w^{\prime}\right)_{a b} \neq \emptyset$.

The following two lemmas relate outcomes of W-monotone, W-tie-breaking and W-gradual weighed tournament correspondence at an elementary change of weighed tournaments $\left(w, w^{\prime}\right)$ from $a b$ to $b a$. Lemma 3 shows that there are no outcome at $w$ ordering $b$ above $a$ precisely when there are outcomes at $w^{\prime}$ ordering $a$ above $b$ and vice versa. In Lemma 4 it is shown that if there are outcomes at $w$ ordering $b$ above $a$, then these form the outcomes at $w^{\prime}$.

Lemma 3 Let $\Phi$ be a W-monotone, W-tie-breaking, and W-gradual weighed tournament correspondence. Let tournament pair $\left(w, w^{\prime}\right)$ form an elementary change in $2 \mathbb{W}$ from $a b$ to ba. Then, either $\Phi(w)_{b a} \neq \emptyset$ or $\Phi\left(w^{\prime}\right)_{a b} \neq \emptyset$.

Proof Consider a tournament correspondence $\Phi$, weighed tournaments $w$ and $w^{\prime}$, and alternatives $a$ and $b$ as in the formulation of the Lemma. We first show that not both $\Phi(w)_{b a} \neq \varnothing$ and $\Phi\left(w^{\prime}\right)_{a b} \neq \varnothing$. On the contrary suppose both $\Phi(w)_{b a} \neq \varnothing$ and $\Phi\left(w^{\prime}\right)_{a b} \neq \varnothing$. We deduce a contradiction. As $\left(w, w^{\prime}\right)$ forms an elementary change from $a b$ to $b a$ and $\Phi(w)_{b a} \neq \emptyset$, W-monotonocity implies $\Phi(w)_{b a} \subseteq \Phi\left(w^{\prime}\right) \subseteq \Phi(w)$. So, $\Phi\left(w^{\prime}\right)_{b a} \neq \varnothing$. Also, $\left(w^{\prime}, w\right)$ forms an elementary change from $b a$ to $a b$ and $\Phi\left(w^{\prime}\right)_{a b} \neq \emptyset$. Therefore, W-monotonocity implies $\Phi\left(w^{\prime}\right)_{a b} \subseteq \Phi(w) \subseteq \Phi\left(w^{\prime}\right)$. So, $\Phi(w)_{a b} \neq \emptyset$. All in all we have that all four $\Phi(w)_{b a}, \Phi\left(w^{\prime}\right)_{b a}, \Phi\left(w^{\prime}\right)_{a b}$, and $\Phi(w)_{a b}$ are non-empty which contradicts the W-tie-breaking.

Next we show that $\Phi(w)_{b a} \neq \emptyset$ or $\Phi\left(w^{\prime}\right)_{a b} \neq \emptyset$. Suppose $\Phi(w)_{b a}=\emptyset$. It is sufficient to show that $\Phi\left(w^{\prime}\right)_{a b} \neq \emptyset$. Since $\left(w, w^{\prime}\right)$ forms an elementary change from $a b$ to $b a$ and $\Phi(w)_{b a}=\emptyset, \mathrm{W}$-graduality implies $\Phi\left(w^{\prime}\right)_{a b} \neq \emptyset$.

Lemma 4 Let $\Phi$ be a W-monotone, W-tie-breaking, and W-gradual weighed tournament correspondence. Let weighed tournament pair $\left(w, w^{\prime}\right)$ form an elementary change in $2 \mathbb{W}$ from $a b$ to ba. Then $\Phi(w)_{b a} \neq \emptyset$ implies $\Phi(w)_{b a}=\Phi\left(w^{\prime}\right)$. 
Proof Consider a tournament correspondence $\Phi$, weighed tournaments $w$ and $w^{\prime}$, and alternatives $a$ and $b$ as in the formulation of the Lemma. Let $\Phi(w)_{b a} \neq \emptyset$. It is sufficient to show that (i) $\Phi(w)_{b a} \subseteq \Phi\left(w^{\prime}\right)$, and (ii) $\Phi\left(w^{\prime}\right) \subseteq \Phi(w)_{b a}$. As $\left(w, w^{\prime}\right)$ forms an elementary change from $a b$ to $b a$, W-monotone implies

$$
\Phi(w)_{b a} \subseteq \Phi\left(w^{\prime}\right) \subseteq \Phi(w)
$$

The former inclusion proves (i). To prove the inclusion at (ii), note that because of $\Phi(w)_{b a} \neq \emptyset$, Lemma 3 implies $\Phi\left(w^{\prime}\right)_{a b}=\emptyset$. So, $\Phi\left(w^{\prime}\right)=\Phi\left(w^{\prime}\right)_{b a}$. Above we already deduced that $\Phi\left(w^{\prime}\right) \subseteq \Phi(w)$. So, $\Phi\left(w^{\prime}\right)_{b a} \subseteq \Phi(w)_{b a}$. Hence, $\Phi\left(w^{\prime}\right)=$ $\Phi\left(w^{\prime}\right)_{b a} \subseteq \Phi(w)_{b a}$. This proves the inclusion at (ii).

Lemma 5 Let $\Phi$ be a $W$-unbiased, W-monotone, $W$-tie-breaking tournament, and $W$ gradual weighed tournament correspondence. Let $w$ be a weighed tournament in $2 \mathbb{W}$, such that its associated tournament, say $T_{w}$, is contained in some linear order. Then

$$
\Phi(w)=\bigcap_{x y \in T_{w}} \mathbb{L}_{x y}=\left\{R \in \mathbb{L}: T_{w} \subseteq R\right\} .
$$

Proof First we prove the second equation. For all $x y$ in $T_{w}$ and all linear orders $R$ such that $T_{w} \subseteq R$ we have $x y$ is in $R$. Therefore, all $R$, with $T_{w} \subseteq R$, are in $\mathbb{L}_{x y}$. This proves that $\left\{R \in \mathbb{L}: T_{w} \subseteq R\right\} \subseteq \bigcap_{x y \in T_{w}} \mathbb{L}_{x y}$. To prove $\bigcap_{x y \in T_{w}} \mathbb{L}_{x y} \subseteq\left\{R \in \mathbb{L}: T_{w} \subseteq R\right\}$, let $R^{\prime}$ be in $\bigcap_{x y \in T_{w}} \mathbb{L}_{x y}$. It is sufficient to prove that $T_{w} \subseteq R^{\prime}$. But by the choice of $R^{\prime}$ we have that $R^{\prime}$ contains all $x y$ which are in $T_{w}$. So, obviously $T_{w} \subseteq R^{\prime}$. This proves the second equation.

To prove the first equation for integers $k \geq 0$ let $\mathcal{P}(k)$ denote the following statement:

For weighed tournaments $v \in 2 \mathbb{W}$, with associated tournament $T_{v}$, such that

$\left|T_{v}\right| \leq k$ and $T_{v} \subseteq R$ for some linear orders $R, \Phi(v)=\bigcap_{x y \in T_{v}} \mathbb{L}_{x y}$.

It is sufficient to prove $\mathcal{P}(k)$ for all integers $k \geq 0$. The proof is done by induction on $k$.

Basis: $k=0$ Let $v$ be a weighed tournament in $2 \mathbb{W}$ such that its associated tournament $T_{v}$ has cardinality zero. This means $T_{v}$ is empty. So, $\bigcap_{x y \in T_{v}} \mathbb{L}_{x y}=\bigcap_{x y \in \emptyset} \mathbb{L}_{x y}=$ $\mathbb{L}$. As $T_{v}$ is empty, $v=\mathbf{0}$. As $\Phi$ is unbiased then $\Phi(v)=\Phi(\mathbf{0})=\mathbb{L}$, which proves the basis.

Induction step Let $\mathcal{P}(k)$, and $v$ be a weighed tournament in $2 \mathbb{W}$, such that its associated tournament $T_{v}$ is contained in a linear order $R^{v}$, and $\left|T_{v}\right|=k+1$. It is sufficient to prove that

$$
\Phi(v)=\bigcap_{x y \in T_{v}} \mathbb{L}_{x y} .
$$

Let $a b \in T_{v}$ and $v_{a b}=2 \cdot l$ for some integer $l \geq 1$. For some linear order $R^{a b}$, at which $a$ is consecutively ordered above $b$, and for some weighed tournament $w^{0}$ we have $w=w^{0}+l \cdot \overline{r^{a b, R^{a b}}}$. For all $t \in\{0,1, \ldots, l\}$ let $v^{t}=w^{0}+t \cdot \overline{r^{a b, R^{a b}}}$. Then $v^{l}=v$ and $\left(v^{t+1}, v^{t}\right)$ form an elementary change from $b a$ to $a b$ in $2 \mathbb{W}$ for all 
$t \in\{0,1, \ldots l-1\}$. Also for $t \in\{0,1, \ldots l-1\}$ the associated tournaments to $v^{t}$ we have $T_{v^{t}} \subseteq T_{v^{t+1}} \subseteq T_{v} \subseteq R^{v}$. In particular $T_{v^{0}} \subseteq R^{v}$. As by construction $T_{v^{0}}=T_{v} \backslash\{a b\}$ it follows that $\left|T_{v^{0}}\right|=k$. Hence, the induction hypothesis $\mathcal{P}(k)$ implies

$$
\Phi\left(v^{0}\right)=\bigcap_{x y \in T_{v^{0}}} \mathbb{L}_{x y} .
$$

Using the second equation we have

$$
\Phi\left(v^{0}\right)=\left\{R \in \mathbb{L}: T_{v^{0}} \subseteq R\right\}
$$

As $T_{v^{0}} \subseteq T_{v} \subseteq R^{v}$, this means that $R^{v} \in \Phi\left(v^{0}\right)$. As $a b \in T_{v}$ we have $\Phi\left(v^{0}\right)_{a b} \neq \emptyset$. Now by Lemma 4

$$
\begin{aligned}
\Phi\left(v^{1}\right) & =\Phi\left(v^{0}\right)_{a b}=\left(\bigcap_{x y \in T_{v^{0}}} \mathbb{L}_{x y}\right)_{a b} \\
& =\left(\bigcap_{x y \in T_{v^{0}}} \mathbb{L}_{x y}\right) \cap \mathbb{L}_{a b} \\
& =\bigcap_{x y \in T_{v^{1}}} \mathbb{L}_{x y}
\end{aligned}
$$

As $\Phi\left(v^{1}\right)=\Phi\left(v^{0}\right)_{a b}$, it follows that $\Phi\left(v^{1}\right)_{a b} \neq \emptyset$. Hence, by Lemma 4 we have

$$
\begin{aligned}
\Phi\left(v^{2}\right) & =\Phi\left(v^{1}\right)_{a b}=\left(\bigcap_{x y \in T_{v^{1}}} \mathbb{L}_{x y}\right)_{a b} \\
& =\bigcap_{x y \in T_{v^{2}}} \mathbb{L}_{x y} .
\end{aligned}
$$

Repeating this reasoning yields that $\Phi\left(v^{l}\right)=\bigcap_{x y \in T_{v}} \mathbb{L}_{x y}$ and as $v=v^{l}$ we have $\Phi(v)=\bigcap_{x y \in T_{v}} \mathbb{L}_{x y}$.

\section{Characterization}

Lemma 1 shows that Kemeny rule is unbiased, monotone, weighed tournamental, strongly tie-breaking, and strongly gradual. Theorem 1 shows that it is the only rule which satisfies these conditions.

Theorem 1 Kemeny rule is the only rule that is unbiased, monotone, weighed tournamental, strongly tie-breaking, and strongly gradual. 
Proof By Lemma 1 the Kemeny rule satisfies these five conditions. In order to prove that it is the only rule, it is sufficient to prove that any two rules satisfying these five conditions are equal. To that end, let $\varphi^{1}$ and $\varphi^{2}$ denote rules satisfying these five conditions. It is sufficient to show that $\varphi^{1}=\varphi^{2}$. Let $\Phi^{1}$ and $\Phi^{2}$ represent the weighed tournament correspondences associated to $\varphi^{1}$ and $\varphi^{2}$ respectively. By Lemma 2 we have that $\Phi^{1}$ and $\Phi^{2}$ are both W-unbiased, W-monotone, W-tie-breaking and Wgradual. Note that it is sufficient to prove that $\Phi^{1}=\Phi^{2}$. Therefore, for all integers $k \geq 0$ it is sufficient to prove statement $\mathcal{Q}(k)$ :

$$
\Phi^{1}(w)=\Phi^{2}(W) \text { for all weighed tournaments } w \in 2 \mathbb{W} \text {, with } \sum_{x y \in A \times A} w_{x y} \leq 2 k \text {. }
$$

We prove this by induction on $k$.

Basis: $k=0$. Then all entries $w_{x y}$ in $w$ are zero. So, $w=\mathbf{0}$ and by $\mathrm{W}$-unbiasedness of $\Phi^{1}$ and $\Phi^{2}$ it follows that $\Phi^{1}(w)=\Phi^{2}(w)=\mathbb{L}$.

Induction step: Assume the induction hypothesis $\mathcal{Q}(k)$. In order to prove $\mathcal{Q}(k+1)$ let $w$ be a weighed tournament in $2 \mathbb{W}$ such that $\sum_{x y \in A \times A} w_{x y}=2(k+1)$. For reasons of symmetry it is sufficient to prove $\Phi^{1}(w) \subseteq \Phi^{2}(W)$. Take $R \in \Phi^{1}(w)$. It is sufficient to prove that $R \in \Phi^{2}(W)$. Consider $T_{w}$ the tournament associated to $w$. We distinguish the following two cases.

Case $1 T_{w} \subseteq R$. Then by Lemma $5 \Phi^{1}(w)=\Phi^{2}(w)=\bigcap_{x y \in T_{w}} \mathbb{L}_{x y}$.

Case 2 There are $a b \in T_{w}$ with $b a \in R$. So, $w_{a b}>0$. Consider $w^{\prime} \in 2 \mathbb{W}$, with $w_{x y}=w_{x y}^{\prime}$ for all $x y \neq a b$, and $w_{a b}^{\prime}=w_{a b}-2$. Then $\left(w, w^{\prime}\right)$ is an elementary change from $a b$ to $b a$ and $\sum_{x y \in A \times A} w_{x y}^{\prime}=2 k$. The induction hypothesis therefore implies that

$$
\Phi^{1}\left(w^{\prime}\right)=\Phi^{2}\left(w^{\prime}\right)
$$

As $R \in \Phi^{1}(w)_{b a}$ Lemma 4 implies

$$
\Phi^{1}(w)_{b a}=\Phi^{1}\left(w^{\prime}\right) .
$$

Combining the latter two equations yields

$$
\Phi^{2}\left(w^{\prime}\right)=\Phi^{1}\left(w^{\prime}\right)=\Phi^{1}(w)_{b a} .
$$

Therefore, $\Phi^{2}\left(w^{\prime}\right)_{a b}=\emptyset$. Applying Lemma 3 to $\Phi^{2}$ implies $\Phi^{2}(w)_{b a} \neq \emptyset$. So, by Lemma 4 this yields

$$
\Phi^{2}(w)_{b a}=\Phi^{2}\left(w^{\prime}\right) .
$$

Using the four latter exposed equations yields $R \in \Phi^{1}(w)_{b a}=\Phi^{1}\left(w^{\prime}\right)=\Phi^{2}\left(w^{\prime}\right)=$ $\Phi^{2}(w)_{b a}$. Hence, $R \in \Phi^{2}(w)$.

\section{Discussion}

This paper provides a new characterization of the Kemeny preference rule with logically independent conditions including monotonicity. In relation to the previous 
characterization, i.e., Can and Storcken (2013), the Kemeny rule is shown to be update monotone, Pareto optimal, consistent and pairwise, while this paper proves the Kemeny rule to be unbiased, update monotone, weighed tournamental, strongly tie-breaking and strongly gradual. It should be noted that being unbiased follows from being neutral. On the other hand, being neutral, consistent, and pairwise together imply being weighed tournamental. In addition this result enables a comparison between Borda and Kemeny rules, which we dwell upon in Sect. 6.1.

As discussed in the introduction, despite being closely related to the Borda choice rule (and in general scoring rules), the Kemeny rule has distinct features, e.g., monotonicity. In fact, as laid out in the section below, Borda (as a preference rule) shares all the features discussed in our paper, except the monotonicity condition. Therefore, our result exposes how closely related these two concepts are, which opens up further questions on how to extend the results of Doğan and Koray (2015) for domains on which scoring (preference) rules can also be monotonic.

Our results also differ from Can and Storcken (2013) by proposing two new conditions, i.e., being strongly tie-breaking and strongly gradual, which also enables a comparison with the characterization of the Slater rule Can et al. (2021). This paper shows that the Kemeny rule is the only rule that is unbiased, monotone, weighed tournamental, strongly tie-breaking, strongly gradual. Can et al. (2021) shows that Slater rule is the only rule that is unbiased, monotone, tournamental, tie-breaking and gradual. Therefore, we also strengthened their result by imposing being strongly gradual. Hence, we catch the similarities between the two well-known rules, the Slater and the Kemeny rules, while reducing the difference between the two to a matter of tie-breaking and weighing the tournaments.

In what follows, we first show how these two preference rules, i.e., the Borda and the Kemeny preference rules relate to one another axiomatically. Thereafter, we show how our results relate to those of Can et al. (2021) regarding the Slater preference rule. We conclude the paper by showing the logical independence of the characterizing conditions laid out in Sect. 6.3.

\subsection{Relation to the Borda preference rule}

We define the Borda preference rule as follows: given a profile $p$, let $\varphi^{B}(p)$ assign all the linear orders which are the transitive closures of the partial order induced by the Borda score of alternatives in $p$. That is, the Borda rule ranks the alternatives based on the number of alternatives that they beat in each individual preference in total. Formally:

$$
\varphi^{B}(p)=\{R \in \mathbb{L}: B(p) \subseteq R\},
$$

where the partial order $B(p)=\{a b \in A \times A: B \operatorname{score}(a, p)>B$ score $(b, p)\}$ and $\operatorname{Bscore}(a, p)=\sum_{i \in N}|\{x \in A: a x \in p(i)\}|$.

Borda preferene rule seems to share many features with those of the Kemeny rule's. In fact it only fails monotonicity among the characterizing conditions of the Kemeny rule. Below we provide an example. 
Example 2 To show that $\varphi^{B}$ is not monotone, consider the preference profile $p=$ $(a b c, b c a, c a b)$ and $q=(b a c, b c a, c a b)$. It can be verified that $B \operatorname{score}(x, p)=3$ for all $x \in A$ and $\operatorname{Bscore}(a, q)=2, \operatorname{Score}(b, q)=4$, and $\operatorname{Score}(c, q)=3$. Therefore, $\varphi^{B}(p)=\mathbb{L}$ and $\varphi^{B}(q)=\{b c a\}$. Note that $(p, q)$ forms an elementary change from $a b$ to $b a$, however $\varphi^{B}(p)_{b a} \nsubseteq \varphi^{B}(q)$, which violates being monotone.

Proposition 1 Borda preference rule is unbiased, weighed tournamental, strongly tiebreaking, and strongly gradual.

Proof It is straightforward to see that the Borda preference rule is unbiased.

To show that it is weighed tournamental, consider any two profiles $p \in L^{N_{1}}$ and $q \in L^{N_{2}}$ such that $\bar{p}=k \bar{q}$ for some $k$. Note that, the Borda score of an alternative $x$ at a profile $p \in \mathbb{L}(A)^{N}$ is defined by

$$
\operatorname{Bscore}(a, p)=\sum_{i \in N}|\{x \in A: a x \in p(i)\}| .
$$

which is equvalent to,

$$
\operatorname{Bscore}(a, p)=\sum_{x \in A}|\{i \in N: a x \in p(i)\}| .
$$

and that

$$
|\{i \in N: a x \in p(i)\}|=\bar{p}_{a x}+\frac{1}{2}\left(|N|-\max \left\{\bar{p}_{a x}, \bar{p}_{x a}\right\}\right)
$$

Therefore,

$$
\begin{aligned}
\operatorname{Bscore}(a, p) & =\sum_{x \in A}|\{i \in N: a x \in p(i)\}| \\
& =\sum_{x \in A}\left(\bar{p}_{a x}+\frac{1}{2}\left(|N|-\max \left\{\bar{p}_{a x}, \bar{p}_{x a}\right\}\right)\right) \\
& =\frac{1}{2}|N| \cdot|A|+\sum_{x \in A}\left(\bar{p}_{a x}-\frac{1}{2} \max \left\{\bar{p}_{a x}, \bar{p}_{x a}\right\}\right) .
\end{aligned}
$$

By the latter equality it follows that the relative Bscores will not change if $\bar{p}=k \bar{q}$ for $p \in \mathbb{L}(A)^{N_{1}}$ and $q \in \mathbb{L}(A)^{N_{2}}$ as

$$
\begin{aligned}
& \operatorname{Bscore}(a, q)>\operatorname{Bscore}(b, q) \\
& \Longleftrightarrow \frac{1}{2}\left|N_{2}\right| \cdot|A|+\sum_{x \in A}\left(\bar{q}_{a x}-\frac{1}{2} \max \left\{\bar{q}_{a x}, \bar{q}_{x a}\right\}\right) \\
& >\frac{1}{2}\left|N_{2}\right| \cdot|A|+\sum_{x \in A}\left(\bar{q}_{a x}-\frac{1}{2} \max \left\{\bar{q}_{a x}, \bar{q}_{x a}\right\}\right)
\end{aligned}
$$




$$
\begin{aligned}
\Longleftrightarrow & \frac{1}{2}\left|N_{1}\right| \cdot|A|+k \cdot \sum_{x \in A}\left(\bar{q}_{a x}-\frac{1}{2} \max \left\{\bar{q}_{a x}, \bar{q}_{x a}\right\}\right) \\
> & \frac{1}{2}\left|N_{1}\right| \cdot|A|+k \cdot \sum_{x \in A}\left(\bar{q}_{a x}-\frac{1}{2} \max \left\{\bar{q}_{a x}, \bar{q}_{x a}\right\}\right) \\
\Longleftrightarrow & \frac{1}{2}\left|N_{1}\right| \cdot|A|+\sum_{x \in A}\left(\bar{p}_{a x}-\frac{1}{2} \max \left\{\bar{p}_{a x}, \bar{p}_{x a}\right\}\right) \\
& >\frac{1}{2}\left|N_{1}\right| \cdot|A|+\sum_{x \in A}\left(\bar{p}_{a x}-\frac{1}{2} \max \left\{\bar{p}_{a x}, x a\right\}\right) \\
\Longleftrightarrow & B \operatorname{score}(a, p)>B \operatorname{score}(b, p) .
\end{aligned}
$$

In words Bscores at $p$ are obtained from those at $q$ by a monotone transformation. This proves that Borda preference rule is weighed tournamental.

To show that $\varphi^{B}$ is strongly tie-breaking, let $(p, q)$ forms an elementary change from $a b$ to $b a$. The only effect of an elementary change is that the Bscore of $a$ is reduced by one and the Bscore of $b$ is increased by one. Since $\varphi^{B}(p)_{a b} \neq \emptyset$ and $\varphi^{B}(p)_{b a} \neq \emptyset$, then it must be the case that $B \operatorname{score}(a, p)=B$ score $(b, p)$, therefore at $q$ we have Bscore $(a, q)<B \operatorname{score}(b, q)$, this imples that $\varphi^{B}(q)_{b a} \neq \emptyset$ and $\varphi^{B}(q)_{a b}=\emptyset$.

To show that $\varphi^{B}$ is strongly gradual, let $(p, q)$ forms an elementary change from $a b$ to $b a$, with $|N|$ even. The only effect of an elementary change is that the Bscore of $a$ is reduced by one and the Bscore of $b$ is increased by one. Since $\varphi^{B}(p)_{b a}=\emptyset$, then it must be the case that $B$ score $(a, p)>B$ score $(b, p)$, therefore (as the number of agents are even) at $q$ we have $B \operatorname{score}(a, q) \geq B \operatorname{score}(b, q)$, this imples that $\varphi^{B}(q)_{a b} \neq \emptyset$.

\subsection{Relation to the Slater rule}

The Slater rule assigns to every profile $p$ the linear orders that are closest to the pairwise strict majority tournament. Formally, for any profile $p$ the Slater rule is defined by,

$$
\varphi^{S}(p)=\left\{R \in \mathbb{L}: \delta\left(T_{p}, R\right) \leq \delta\left(T_{p}, R^{\prime}\right) \text { for all } R^{\prime} \in \mathbb{L}\right\} .
$$

In Can et al. (2021) the Slater is characterized by five conditions. Two of these conditions, i.e., being unbiased and monotone, are defined in the same way as in this paper. The other three conditions are as follows:

- Tournamental: $\varphi$ is tournamental if for all profiles $p$ and $q$, with $T_{p}=T_{q}$,

$$
\varphi(p)=\varphi(q)
$$

- Tie-breaking: $\varphi$ is tie-breaking if for all pairs of profiles $(p, q)$ forming an elementary change from $a b$ to $b a$ in $\mathbb{L}^{N}$, with $\bar{p}_{a b}=\bar{p}_{b a}=0$,

$$
\text { if } \varphi(p)_{b a} \neq \emptyset \text {, then } \varphi(q)_{a b}=\emptyset \text {. }
$$


- Gradual: $\varphi$ is gradual if for all pairs of profiles $(p, q)$ forming an elementary change from $a b$ to $b a$ in $\mathbb{L}^{N}$, with $\bar{p}_{a b}=\bar{p}_{b a}=0$,

$$
\text { if } \varphi(q)_{a b}=\emptyset \text {, then } \varphi(p)_{b a} \neq \emptyset \text {. }
$$

The first two conditions in these results are the same, i.e., being unbiased and monotone. For the other conditions, note that if two profiles $p, q$ have the same weighed tournaments $\bar{p}=\bar{q}$, then they also have the same pairwise majority tournaments $T_{p}=T_{q}$ (and not the other way around). Therefore, being tournamental implies being weighed tournamental. While the Slater rule satisfies both, the Kemeny rule is only weighed tournamental. On the other hand, Kemeny rule is strongly tie-breaking, while Slater is not (see the example below). Finally, being strongly gradual is a condition satisfied by both rules (see proposition below).

Example 3 Let $A=\{a, b, c\}$ be the set of alternatives and $N=\{1, \ldots, 12\}$ be the set of agents. Consider the preference profile $p=(a b c, b c a, c a b, a b c, b c a, c a b, a b c$, $b c a, c a b, a b c, b c a, c a b)$. Let $q=(b a c, b c a, c a b, a b c, b c a, c a b, a b c, b c a, c a b$, $a b c, b c a, c a b)$. Note that $(p, q)$ forms an elementary change from $a b$ to $b a$, and $T_{p}=T_{q}=\{a b, b c, c a\}$. Therefore the outcomes of the Slater rule at both $p$ and $q$ are $\varphi^{S}(p)=\varphi^{S}(q)=\{a b c, b c a, c a b\}$. This violates being strongly tie-breaking.

Proposition 2 The Slater satisfies being strongly gradual.

Proof Let $(p, q)$ forms an elementary change from $a b$ to $b a$ in $\mathbb{L}^{N}$, with $|N|$ even. We show that if $\varphi^{S}(p)_{b a}=\emptyset$, then $\varphi^{S}(q)_{a b} \neq \emptyset$.

As $(p, q)$ forms an elementary change from $a b$ to $b a$, three cases are possible:

- $T_{p}=T_{q}$. As $\varphi^{S}(p)_{b a}=\emptyset$, then $\varphi^{S}(p)_{a b} \neq \emptyset$. As Slater is tournamental, then $\varphi^{S}(q)_{a b} \neq \emptyset$.

- $T_{p}=T_{q}-\{b a\}$ with $b a \in T_{q}$. As the number of agents are even, this implies that at $p$ we have $\bar{p}_{a b}=\bar{p}_{b a}=0$. Then as Slater is gradual, (the contraposition) implies if $\varphi^{S}(p)_{b a}=\emptyset$, then $\varphi^{S}(q)_{a b} \neq \emptyset$.

- $T_{q}=T_{p}-\{a b\}$ with $a b \in T_{p}$. As the number of agents are even, this implies that at $q$ we have $\bar{q}_{a b}=\bar{q}_{b a}=0$. Note that $(q, p)$ forms an elementary change from $b a$ to $a b$, with $\bar{q}_{a b}=\bar{q}_{b a}=0$. Hence, being gradual implies if $\varphi(p)_{b a}=\emptyset$, then $\varphi(q)_{a b} \neq \emptyset$.

Therefore, in each case if $\varphi^{S}(p)_{b a}=\emptyset$, then $\varphi(q)_{a b} \neq \emptyset$, which completes the proof.

\subsection{Logical independence}

In this section we show the characterizing conditions are logically independent. That is, we find rules satisfying all characterizing conditions but one. Consider the following rules at any profile $p$ in $\mathbb{L}^{N}$ :

- Constant $R$ rule: Given a fixed $R \in \mathbb{L}$, this rule assigns $R$ to all profiles:

$$
\varphi^{R}(p)=\{R\}
$$


- Borda rule: Defined in Sect. 6.1.

- Combination rule: Given a dictator in $N$, e.g., agent 1 , the combination rule becomes dictatorial when there are odd number of agents, and equals the Kemeny rule otherwise.

$$
\varphi^{D \& K}(p)= \begin{cases}\{p(1)\}, & \text { if }|N| \text { is odd } \\ \varphi^{K}(p), & \text { if }|N| \text { is even }\end{cases}
$$

- Indecisive rule: This rule assigns all the linear to all profile,

$$
\varphi^{\mathbb{L}}(p)=\mathbb{L} .
$$

- Weighed Kemeny rule: Given a fixed weight distribution on all pairs of alternatives, this rule assigns the linear orders that are closest to the profile in terms of weighed Kemeny distance.

$$
\varphi^{W}(p)=\left\{R \in \mathbb{L}: W(R, p) \leq W\left(R^{\prime}, p\right) \text { for all } R^{\prime} \in \mathbb{L}\right\}
$$

where $W(R, p)=\sum_{x y \in R} \omega(y x) \cdot \bar{p}_{y x}$ and $\omega(x y)$ are strictly positive real numbers for all pairs $x y$ of distinct alternatives $x$ and $y$. Note that the Kemeny rule is a weighed Kemeny rule where all weights $\omega(x y)$ are equal to each other. For a discussion on weighted Kemeny distances see Can (2014).

Example 4 Let $A=\{a, b, c\}$. Let $\omega(a b)=\omega(b a)=2$ and $\omega(x y)=1$ for all $x y$ other than $a b$ and $b a$. Let $N=\{1, \ldots, 6\}$. Consider the following profiles:

$$
\begin{aligned}
& p=(a b c, a b c, b c a, b c a, c a b, c a b), \\
& q=(b a c, a b c, b c a, b c a, c a b, c a b) .
\end{aligned}
$$

Then, $W(c b a, p)=\omega(a b) \cdot \bar{p}_{a b}+\omega(b c) \cdot \bar{p}_{b c}+\omega(a c) \cdot \bar{p}_{a c}=2 \cdot 4+1 \cdot 4+1 \cdot 0=12$. Similarly we have $W(a b c, p)=4, W(a c b, p)=8, W(c a b, p)=4, W(b c a, p)=8$, and $W(b a c, p)=12$. For profile $q$ we have $W(c b a, q)=4, W(a b c, q)=4$, $W(a c b, q)=8, W(c a b, q)=4, W(b c a, q)=0$, and $W(b a c, q)=4$. Therefore, $\varphi^{W}(p)=\{a b c, c a b\}$ and $\varphi^{W}(q)=\{b c a\}$.

The rules we have defined above complete the logical independence of the characterizing conditions in Sect. 3. The constant $R$ rule $\left(\varphi^{R}\right)$ satisfies all, but being unbiased. The Borda rule $\left(\varphi^{B}\right)$ satisfies all, but being monotone. The combination rule $\left(\varphi^{D \& K}\right)$ satisfies all, but being weighed tournamental. The indecisive rule $\left(\varphi^{\mathbb{L}}\right)$ satisfies all, but being strongly tie-breaking. The weighed Kemeny rule $\left(\varphi^{W}\right)$ satisfies all, but being strongly gradual. Table 1 summarizes these results and shows the conditions are independent.

In the following list of explanations, the list number corresponds to the superscripts in Table 1.

1. These are obvious or by construction of the rule.

2. In Example 2 we showed that $\varphi^{B}$ is not monotone.

3. In Sect. 6.1 we showed that $\varphi^{B}$ is weighed tournamental. 
Table 1 Logical independence of the conditions

\begin{tabular}{|c|c|c|c|c|c|}
\hline Condition & $\varphi^{R}$ & $\varphi^{B}$ & $\varphi^{D \& K}$ & $\varphi^{\mathbb{L}}$ & $\varphi^{W}$ \\
\hline Unbiased & -1 & +1 & $++^{1}$ & $++^{1}$ & $+^{1}$ \\
\hline Monotone & $+^{1}$ & -2 & $+{ }^{6}$ & $+^{1}$ & $+^{8}$ \\
\hline Weighed tournamental & $+^{1}$ & $+^{3}$ & -7 & $+^{1}$ & $+^{1}$ \\
\hline Strongly tie-breaking & $+^{1}$ & $+^{4}$ & $+^{1}$ & -1 & $+{ }^{9}$ \\
\hline Strongly gradual & $++^{1}$ & $+^{5}$ & $++^{1}$ & $+^{1}$ & $-{ }^{10}$ \\
\hline
\end{tabular}

+: satisfied, - : unsatisfied

4. In Sect. 6.1 we showed that $\varphi^{B}$ is strongly tie-breaking.

5. In Sect. 6.1 we showed that $\varphi^{B}$ is strongly gradual.

6. In case the number of agents is even being monotone follows as the Kemeny rule is monotone by Lemma 1 . In case the number of agents is odd, let $\varphi^{D \& K}(p)_{b a}$ be non-empty. It is sufficient to prove $\varphi^{D \& K}(p)_{b a} \subseteq \varphi^{D \& K}(q) \subseteq \varphi^{D \& K}(p)$. As $\varphi^{D \& K}(p)_{b a}$ is non-empty, $\varphi^{D \& K}(p)_{b a}=\{p(1)\}$ and $b a \in p(1)$. So, the agent that does change the preference going to profile $q$ is not agent 1 . So, $\varphi^{D \& K}(p)_{b a}=$ $\varphi^{D \& K}(q)=\varphi^{D \& K}(p)=\{p(1)\}$, which proves the inclusions.

7. At the profile $p=(b a c, a c b, c b a)$ there are three agents. So, $\varphi^{D \& K}(p)=\{b a c\}$. At the profile $p+p$, we have an even number of agents. So, $\varphi^{D \& K}(p+p)=$ $\varphi^{K}(p+p)=\{b a c, a c b, c b a\}$. As $2 \cdot \bar{p}=\overline{p+p}$ this difference in outcomes shows that the combination rule is not weighed tournamental.

8. Let $(p, q)$ form an elementary change from $a b$ to $b a$, then for all distinct $x$ and $y$ in $A \backslash\{a, b\} \bar{p}_{x y}=\bar{q}_{x y}$ and we have one of the following three

(a) $\bar{p}_{a b} \geq 2, \bar{q}_{a b}=\bar{p}_{a b}-2$, and $\bar{p}_{b a}=\bar{q}_{b a}=0$,

(b) $\bar{p}_{a b}=1, \bar{q}_{b a}=1$, and $\bar{p}_{b a}=\bar{q}_{a b}=0$,

(c) $\bar{p}_{a b}=\bar{q}_{a b}=0$, and $\bar{p}_{b a}+2=\bar{q}_{b a}$.

Take any $R \in \mathbb{L}$. Then based on whether $R \in \mathbb{L}_{a b}$ or $R \in \mathbb{L}_{b a}$, and the above three cases the value of $W(R, p)-W(R, q)$ is as in the following table:

\begin{tabular}{ccc}
\hline Case & $R \in \mathbb{L}_{a b}$ & $R \in \mathbb{L}_{b a}$ \\
\hline (a) & 0 & $-2 \cdot \omega(a b)$ \\
(b) & $\omega(b a)$ & $-\omega(a b)$ \\
(c) & $2 \cdot \omega(b a)$ & 0 \\
\hline
\end{tabular}

By this table we see that $W(R, p)$ increases by the same value when compared to $W(R, q)$ for all $R \in \mathbb{L}_{a b}$, or $W(R, p)=W(R, q)$ for all $R \in \mathbb{L}_{a b}$. Further, we see that $W(R, p)$ decreases by by the same value when compared to $W(R, q)$ for all $R \in \mathbb{L}_{b a}$, or $W(R, p)=W(R, q)$ for all $R \in \mathbb{L}_{b a}$. As there is either an increase or a decrease we may conclude that if $\varphi^{W}(p)_{b a} \neq \varnothing$, then $\varphi^{W}(q)=\varphi^{W}(p)_{b a}$. By this it follows that the weighed Kemeny rule is monotone. 
9. At 7 we proved $\varphi^{W}(q)=\varphi^{W}(p)_{b a}$ for elementary changes $(p, q)$ from $a b$ to $b a$ at which $\varphi^{W}(p)_{b a} \neq \varnothing$. This implies that the weighed Kemeny rule is strongly tie-breaking.

10. The profiles presented in Example 4 forms an elementary change from $a b$ to $b a$. However, $\varphi^{W}(p)=\{a b c, c a b\}$ and $\varphi^{W}(q)=\{b c a\}$, which violates being strongly gradual.

\section{References}

Borda Jd (1784) Mémoire sur les élections au scrutin. Histoire de l'Academie Royale des Sciences pour 1781 (Paris, 1784)

Can B (2014) Weighted distances between preferences. J Math Econ 51:109-115

Can B, Pourpouneh M, Storcken T (2021) An axiomatic characterization of the Slater rule. Soc Choice Welfare 56(4):835-853

Can B, Storcken T (2013) Update monotone preference rules. Math Soc Sci 65(2):136-149

Conitzer V (2006) Computing Slater rankings using similarities among candidates. In: Proceedings of the National Conference on artificial intelligence, pp 613-619. Menlo Park, CA; Cambridge, MA; London; AAAI Press; MIT Press; 1999

Dodgson C (1876) A method of taking votes on more than two issues: the theory of committees and elections

Doğan B, Koray S (2015) Maskin-monotonic scoring rules. Soc Choice Welfare 44(2):423-432

Elkind E, Faliszewski P, Slinko A (2009) On distance rationalizability of some voting rules. In: Proceedings of the 12th conference on theoretical aspects of rationality and knowledge, pp 108-117

Elkind E, Faliszewski P, Slinko A (2010). On the role of distances in defining voting rules. In: Proceedings of the 9th international conference on autonomous agents and multiagent systems: volume 1, pp 375-382

Endriss U, de Haan R (2015) Complexity of the winner determination problem in judgment aggregation: Kemeny, Slater, Tideman, Young. In: Proceedings of the 2015 international conference on autonomous agents and multiagent systems, pp 117-125

Kemeny J (1959) Mathematics without numbers. Daedalus 88(4):577-591

Klamler C (2004). The Dodgson ranking and its relation to Kemeny's method and Slater's rule. Soc Choice Welfare 23(1): 91-102

Maskin E (1999) Nash equilibrium and welfare optimality. Rev Econ Stud 66(1):23-38

May KO (1952) A set of independent necessary and sufficient conditions for simple majority decision. Econom: J Econom Soc, pp 680-684

McGarvey DC (1953) A theorem on the construction of voting paradoxes. Econom: J Econom Soc, pp $608-610$

Nitzan S (1981) Some measures of closeness to unanimity and their implications. Theor Decis 13(2):129138

Saari DG, Merlin VR (2000) A geometric examination of Kemeny's rule. Social Choice Welfare 17(3):403438

Slater P (1961) Inconsistencies in a schedule of paired comparisons. Biometrika 48(3/4):303-312

Young HP (1974) An axiomatization of Borda's rule. J Econ Theory 9(1):43-52

Young HP, Levenglick A (1978) A consistent extension of Condorcet's election principle. SIAM J Appl Math 35(2):285-300

Publisher's Note Springer Nature remains neutral with regard to jurisdictional claims in published maps and institutional affiliations. 